\title{
ESTUDO DA PRODUÇÃO DE BIOSSURFACTANTE POR Bacillus subtilis, À PARTIR DA COMBINAÇÃO DA BORRA DE REFINO DE ÓLEO VEGETAL E GLICOSE
}

\author{
Márcio Costa Pinto da Silva1; Edna dos Santos Almeida2; Érika Durão Vieira ${ }^{3}$ \\ ${ }^{1}$ Av. Orlando Gomes, 1845 - Piatã, Salvador/BA; marcio.eng.seg2@gmail.com \\ ${ }^{2}$ Av. Orlando Gomes, 1845 - Piatã, Salvador/BA \\ ${ }^{3}$ Av. Orlando Gomes, 1845 - Piatã, Salvador/BA
}

Resumo: O objetivo deste artigo é a produção de biossurfactante utilizando como substrato um resíduo originado no processo de refino de óleo de soja e algodão, (borra), combinado com glicose. A metodologia consiste na ativação do Bacillus subtilis e posterior inoculação, em meio de cultura contendo borra e glicose em variações de percentuais em peso, utilizando o planejamento experimental estrela, acrescido de micronutrientes. Esta borra contendo ácidos graxos, triglicerídeos e produtos da degradação do óleo se mostra promissora como substrato para produção de biossurfactantes, o que pode ser evidenciado pelos resultados das análises de colapso da gota e índice de emulsificação do biossurfactante produzido, atingindo $77 \%$ de eficiência, em valores normalizados, com relação ao surfactante industrial.

Palavras-Chave: Óleo Vegetal; Borra; Biossurfactante; Bacillus subtilis.

\section{STUDY BIOSAFACTANT PRODUCTION, BY Bacillus subtillis, FROM THE COMBINATION OF VEGETABLE OIL REFINING LEES AND GLUCOSE}

\begin{abstract}
The objective of this paper is the production of biosurfactant using as a substrate a residue originated from the refining process of soy and cotton oil (sludge) combined with glucose. The methodology consists of activation of Bacillus subtilis and subsequent inoculation in culture medium containing sludge and glucose in percentage changes in weight, using the experimental star design, plus micronutrients. This sludge containing fatty acids, triglycerides and oil degradation products is promising as a substrate for biosurfactant production, which can be evidenced by the results of drop collapse analysis and emulsification index of the biosurfactant produced, reaching $77 \%$ efficiency, normalized values for industrial surfactant.
\end{abstract}

Keywords: Vegetable oil; Sludge; Biosurfactant; Bacillus subtilis. 


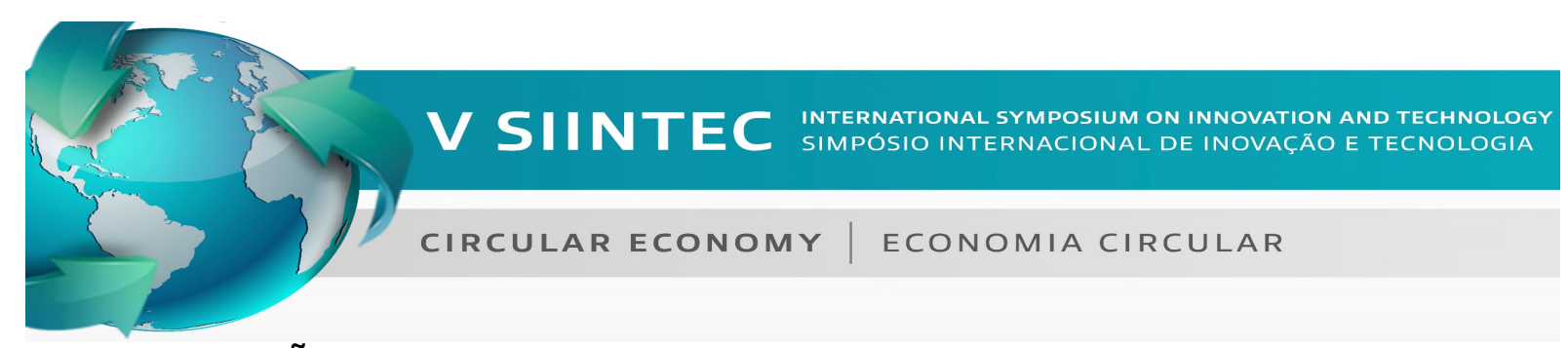

1. INTRODUÇÃO

Os biossurfactantes são substâncias sintetizadas por microrganismos e tem um caráter anfipático, contendo uma parte hidrofílica e outra hidrofóbica. São compostos ativos, com capacidade de reduzir as tensões superficial, possuindo baixa toxicidade, alta biodegradabilidade, com produção a partir de fontes renováveis [1].

Devido à vantagem de serem mais biodegradáveis quando comparados com os surfactantes de origem química, eles são mais apropriados para serem empregados em aplicações, como: biorremediação e dispersão no derramamento de óleos; recuperação melhorada do petróleo; remoção de resíduos de óleo em tanques de estocagem; formulações de herbicidas e pesticidas; aplicação farmacêutica; utilizados em produtos de higiene e cosméticos, devido à sua compatibilidade com a pele $[2,3]$.

Contudo, a produção de biossurfactantes é limitada devido ao seu alto custo, agregada a uma baixa produtividade e uso de substratos caros. Os metabólitos produzidos à partir de substratos baratos, renováveis e através de processos economicamente viáveis permitem diminuir os custos de produção dos biossurfactantes [1].

A viabilidade econômica da produção de biossurfactantes a nível industrial está diretamente ligada ao custo do meio de cultura, (substrato). Neste sentido, a utilização de resíduos ou subprodutos industriais são uma excelente alternativa para a redução de custos na produção de biossurfactantes. Principalmente se este resíduo possuir uma grande fonte de carbono que é o principal metabólito para o crescimento de microorganismos [4].

Um potencial resíduo para servir como meio de cultura com este fim é a borra gerada na etapa de neutralização do refino químico de óleos vegetais. Esta borra é um dos sub produtos da reação do óleo com hidróxido de sódio e é separada na centrifugação. A borra contém ácidos graxos, (fonte de carbono), em valores variando entre 35 a 50\%, sais de sódio, água, triglicerídeos, material saponificável e produtos da degradação do óleo [5]. Este resíduo alcança baixo preço de mercado.

Assim, o objetivo deste trabalho é avaliar o potencial de reaproveitamento desta borra, originada da mistura do refino de óleo de soja e algodão, associado ao substrato glicose, para uso como meio de cultura, visando a produção de biossurfactantes.

\section{METODOLOGIA}

A metodologia constituiu na ativação do bacillus subtilis e posterior inoculação, em meio de cultura contendo borra e glicose em variações de percentuais em peso, utilizando o planejamento experimental estrela, acrescido de micronutrientes e as análises preliminares da evidência da produção de biosurfactante.

\subsection{Ativação e Conservação do Micro organismo}


A cepa indicada para crescimento e produção do biossurfactante em substrato oleaginoso é a do Bacillus subtilis que produz a Surfactina. Foram seguidos os procedimentos para conservação e propagação do microrganismo descrito a seguir.

Materiais: Acessórios: 7 Alças, 10 alças de Drigalski, 1 caixa de ponteiras $1 \mathrm{ml}$, 1 caixa de ponteiras $10 \mathrm{ml}, 60$ criotubos, 1 Pipeta automática de $1 \mathrm{ml}$, 1 Pipeta automática de $10 \mathrm{ml}, 10$ placas de petri descartáveis, 20 tubos de ensaio com 9,2 ml de água destilada, 4 erlenmeyer de $250 \mathrm{ml}, 2$ erlenmeyer de $500 \mathrm{ml}$.

Soluções: Meio de cultura - peptona animal 5,00\% (m/v), extrato de carne $1,50 \%(\mathrm{~m} / \mathrm{v})$, extrato de levedura $1,50 \%(\mathrm{~m} / \mathrm{v})$, cloreto de sódio $\mathrm{NaCl} 5,00 \%(\mathrm{~m} / \mathrm{v})$. Meio para plaqueamento - ágar, solução glicerol $40 \%$.

Preparo dos tubos slant: fez uma solução só com ágar em um erlenmeyer de $250 \mathrm{ml}$ que foi autoclavado. Em seguida colocou-se nos tubos e deixou em repouso com inclinação de $45^{\circ}$.

Preparo do pré inoculo: retirou-se do criotubo a bactéria e inoculou no meio preparado (meio 1) e no tubo slant (procedimento feito em capela de fluxo laminar). Levou-se o meio 1 para a incubadora a $37^{\circ} \mathrm{C}$ e o tubo slant para a estufa a $37^{\circ}$.

Preparo do plaqueamento: com o erlenmeyer de $500 \mathrm{ml}$ com ágar já autoclavado, a solução foi levada ao micro-ondas e depois distribuida nas placas.

Preparo do primeiro repique: colocou-se $10 \mathrm{ml}$ do meio 1 para o meio 2. Em seguida, retirou-se $1 \mathrm{ml}$ do meio 2 para o primeiro tubo de ensaio e diluiu dez vezes, fazendo o plaqueamento nas últimas 5 placas com $200 \mu$ ldos tubos. Levou-se os dois meios para incubadora a $37^{\circ} \mathrm{C}$.

Preparo do segundo repique: colocou-se $10 \mathrm{ml}$ do meio 2 para o meio 3 . Em seguida retirou-se $1 \mathrm{ml}$ do meio 3 para o primeiro tubo e diluiu-se dez vezes, fez o plaqueamento nas últimas 5 placas com $200 \mu \mathrm{l}$ dos tubos. $O$ meio 3 e $110 \mathrm{ml}$ da solução de glicerol foram adicionados no erlenmeyer de $500 \mathrm{ml}$.

Foi realizado a densidade ótica do meio 1 e 2 (não congelados) no espectrofotômetro, utilizando água como referência, diluindo os meios em 5,10 e 15 vezes. Ao final essa cultura foi transferida para 60 criotubos com congelamento rápido de $\mathrm{CO}_{2} \mathrm{a}-80^{\circ} \mathrm{C}$ em etanol e conservados em temperatura abaixo de $0^{\circ} \mathrm{C}$.

\subsection{Propagação dos Micro Organismos}

A propagação ocorreu em meio TSB, (Caldo Tripticaseína de Soja), através de inoculação em Erlenmeyer, previamente preparado com o meio na proporção de $20 \mathrm{~g}$ do meio por litro. Transferiu-se as cepas do criotubo para tubo de ensaio e repicar 10 $\mathrm{ml}$ em $100 \mathrm{ml}$ de 1 a 10 tubos e 5 placas e foram inseridos $110 \mathrm{ml}$ de glicerol $40 \% \mathrm{em}$ $110 \mathrm{ml}$ de cultura fresca do Erlenmeyer, produzindo $220 \mathrm{ml}$ de cultura em glicerol $20 \%$.

\subsection{Ensaios de Fermentação para Produção de Biossurfactantes}

\subsubsection{Melhoramento do meio de cultivo}


O melhoramento do meio de cultivo variando os macros, micronutrientes e indutores, de forma que tenha uma melhor eficiência de produção do biossurfactante. Inicialmente foi inoculado o Bacillus subtilis propagado, conforme descrito acima.

Para enriquecimento do substrato foi utilizado meio basal constituído de ( $\mathrm{g} / \mathrm{L}$ ): MgSO4.7H2O (0.5), KH2PO4 (1.0), NaNO3 (3.0), FeSO4.7H2O (0.01) e acrescidos de extrato de levedura (0.1) [6]. O meio contendo sais de ferro tem um efeito significativo no rendimento da surfactina [7].

$\mathrm{O}$ pH é um fator importante para produção de surfactina. Foi mantido o pH entre 6,7 a 7,2 produz maior quantidade de surfactina $(1,0 \mathrm{~g} / \mathrm{L})$ e temperatura de $37,4 \mathrm{C}$ e velocidade de agitação de 400 rpm [7].

\subsubsection{Combinação das fontes de carbono}

Foram testadas a combinação das fontes de carbono: glicose [8] e borra no fatorial $n=2$, utilizando o planejamento experimental estrela, nas seguintes concentrações (1, 2, 3\% para glicose e 0,1, 2\% para borra).

O planejamento experimental estrela, (Gráfico 1), balizam 8 pontos de experimento e 3 pontos em branco, conforme Tabela 1.

Gráfico 1: Planejamento experimental estrela

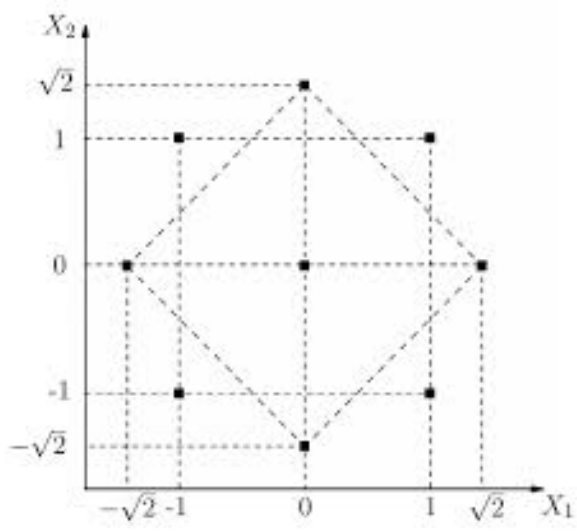

Tabela 1: Relação estrela dos parâmetros de percentual de glicose e borra

$\begin{array}{cccc} & -1 & 0 & 1 \\ \text { Glicose(f1) } & 1 \% & 2 \% & 3 \% \\ \text { Borra (f2) } & 0 \% & 1 \% & 2 \% \\ & & & \\ \text { Pt } & \text { Codificado } & f(1) & f(2) \\ 1 & (-1,-1) & 1 \% & 0 \% \\ 2 & (1,-1) & 3 \% & 0 \% \\ 3 & (-1,1) & 1 \% & 2 \%\end{array}$




$\begin{array}{cccc}4 & (1,1) & 3 \% & 2 \% \\ 5 & (0,0) & 2 \% & 1 \% \\ 6 & (0,0) & 2 \% & 1 \% \\ 7 & (0,0) & 2 \% & 1 \% \\ 8 & (- \text { Rz2,0) } & 0,59 \% & 1 \% \\ 9 & (0,- \text { Rz2) } & 2 \% & 2,41 \% \\ 10 & (\text { Rzz } 20) & 3,41 \% & 1 \% \\ 11 & (0, \text { Rz2 }) & 2 \% & 2,41 \%\end{array}$

\subsection{Produção do biossurfactante:}

Após 24 horas de incubação a 36 C, 200 rpm e razão de aeração de 0,4, definida como a razão do volume do meio de cultivo e volume do frasco [7], foram retiradas as amostras e analisadas: a absorbância e $\mathrm{pH}$ do fermentado e centrifugadas por 20 min. para separação das células e secas em estufa a vácuo a $45^{\circ} \mathrm{C}$ até peso constante [8] sendo analisado a absorbância da suspensão e pesado o sedimentado.

\subsection{Determinação da presença de biossurfactante:}

Os caldos fermentados livres de células foram submetidos às seguintes análises: teste de colapso da gota e índice de emulsificação.

\subsubsection{Teste de colapso da gota}

O teste qualitativo do colapso da gota para detecção da produção de biossurfactantes foi realizada em placas de petri. Cada placa foi untada com $2 \mathrm{~mL}$ de óleo de motor 10 W-40 e colocado 5 gotas do sobrenadantes das amostras 2, 3, 9, 11 e surfactante industrial utilizado como parâmetro. Avaliado de imediato, visualmente. $\mathrm{O}$ resultado foi considerado positivo quando se percebeu, visualmente, a gota colapsada [9].

\subsubsection{Determinação da atividade emulsificante}

A atividade emulsificante foi determinada em tubos de ensaio com tampa de rosca pela adição de $2,0 \mathrm{~mL}$ de tolueno a $3,5 \mathrm{~mL}$ do sobrenadante das amostras 2,3 , 9,11 e o surfactante industrial, que serviu como parâmetro. Misturado em agitador de tubo por 2 min. e deixado em repouso por 24 horas. O índice foi calculado como porcentagem da altura da camada emulsificada $(\mathrm{cm})$ dividida pela altura total da coluna do líquido $(\mathrm{cm})[10]$.

A normalização dos dados foi feita através da ponderação, considerando o resultado da amostra do surfactante industrial como $100 \%$. 


\section{RESULTADOS E DISCUSSÃO}

O teste de colapso da gota é percebido, visualmente: com água e óleo, mantém a imiscibilidade e a tensão superficial, conforme tudo à esquerda da Figura 1 ; com surfactante industrial e óleo há o colapso da gota, conforme visto no tubo à direita da Figura 1.

Figura 1. à esquerda óleo e água; à direita óleo e surfacatante

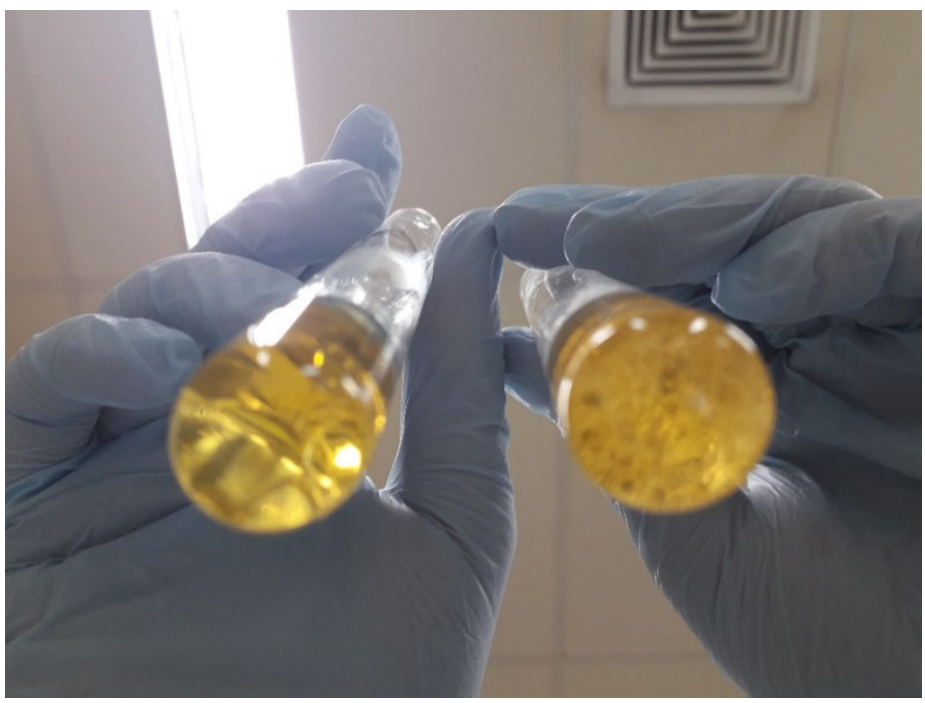

Na figura 2, nas placas de Petri, foram observadas, da esquerda para direita, respectivamente: com água, com surfactante industrial, com amostras 9 e 11. Nas últimas 3 placas há total quebra da tensão superficial, colapsando a gota.

Figura 2. Teste de colapso da gota

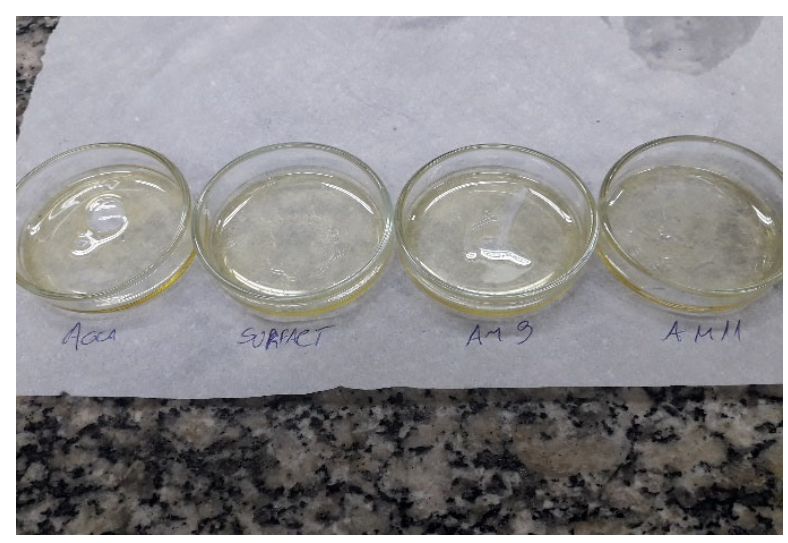

$\mathrm{Na}$ determinação da atividade emulsificante foi feito o teste em duplicata, apresentando resultados nas Tabelas 1 e 2. 
V SIINTEC INTERNATIONAL Symposium ON INNOVATION AND TECHNOLOGY Simpósio internacional de inovação e tecnologia

\begin{tabular}{l|l} 
CIRCULAR ECONOMY & ECONOMIA CIRCULAR
\end{tabular}

Tabela 1. Índice de emulsificação, teste 1

\begin{tabular}{|l|c|c|c|}
\hline Amostras & $\begin{array}{c}\text { Altura camada } \\
\text { emulsificada } \\
(\mathrm{cm})\end{array}$ & $\begin{array}{c}\text { Altura Total } \\
(\mathrm{cm})\end{array}$ & $\%$ \\
\hline $\mathbf{2}$ & 1,3 & 3,5 & 37 \\
\hline $\mathbf{3}$ & 1,2 & 3,4 & 35 \\
\hline $\mathbf{9}$ & 1,4 & 3,8 & 37 \\
\hline $\mathbf{1 1}$ & 1,2 & 3,7 & 32 \\
\hline Surfactante inds & 1,7 & 3,5 & 49 \\
\hline
\end{tabular}

Tabela 2. Índice de emulsificação, teste 2

\begin{tabular}{|l|c|c|c|}
\hline Amostras & $\begin{array}{c}\text { Altura camada } \\
\text { emulsificada } \\
(\mathrm{cm})\end{array}$ & $\begin{array}{c}\text { Altura Total } \\
(\mathrm{cm})\end{array}$ & $\%$ \\
\hline $\mathbf{2}$ & 1,1 & 3,6 & 31 \\
\hline $\mathbf{3}$ & 1,3 & 3,5 & 37 \\
\hline $\mathbf{9}$ & 1,4 & 3,8 & 37 \\
\hline $\mathbf{1 1}$ & 1,3 & 3,5 & 37 \\
\hline Surfactante inds & 1,9 & 3,9 & 49 \\
\hline
\end{tabular}

Em ambos testes a amostra 9 apresentou maior percentual.

\section{CONCLUSÃO}

Do resultado da média das análises em duplicidade foi tirada a conclusão que a amostra 9 contendo $2 \%$ de glicose e $2,41 \%$ de borra foi a que apresentou o maior percentual da camada emulsificante, concluindo pela produção de maior quantidade biossurfactante das amostras analisadas. Comparando ao surfactante industrial, a amostra 9 atingiu $77 \%$ de eficiência, em resultado normalizado, ao surfactante industrial.

\section{Agradecimentos}

Agradeço à FAPESB, bolsa 0232/18, pelo apoio financeiro ao projeto. 


\section{REFERÊNCIAS}

${ }^{1}$ BEZERRA, M. S. Estudo da Produção de biosurfactante sintetizados por Pseudomonas aeruginosa AP029-GVIIA utilizando manipueira como fonte de carbono, UFRN, 2012.

${ }^{2}$ NITSCHKE, M. et al. Structure and Applications of a Rhamnolipid Surfactant Produced in Soybean Oil Waste. APPLIED BIOCHEMISTRY AND BIOTECHNOLOGY, 2010.

3 PIRÔLLO, M. P. S. Estudo da Produção de Biossurfactante utilizando Hidrocarboneto. UNESP, Rio Claro, 2006.

${ }^{4} \mathrm{NITSCHKE}, \mathrm{M}$. et al. Oil wastes as unconventional substrates for rhamnolipid biosurfactant production by Pseudomonas aeruginosa LBI. BIOTECHNOLOGY PROGRESS, 2005.

${ }^{5} \mathrm{BIO}$ ÓLEO Bahia - Indústria de processamento de óleos vegetais $\mathrm{BR} 324$, Feira de Santana - BA, 2018.

6 FARIA, A. F. Produção, Purificação e Caracterização Química de Biossurfactantes Produzidos por Bacillus subtilis em Glicerina Residual, UNICAMP, 2010.

${ }^{7}$ ROCHA, P. M. Produção de surfactina por Bacillus subtilis UFPEDA 438 utilizando melaço da cana como substrato. 2017, RN.

${ }^{8}$ BUENO, S. M. Bactérias produtoras de biossurfactantes: Isolamento, produção, caracterização e comportamento num sistema modelo, 2008, SP.

9 YOUSSEF N. H.; DUNCAN K. E.; NAGLE D. P.; SAVAGE K. N.; KNAPP R. M.; MCINERNEY M. J. Comparison of methods to detect biosurfactant production by diverse microorganisms. J Microbiol Methods, 2004.

${ }^{10}$ BICCA, F. C.; FLECK, L. C.; AYUB, M. A. Z. Production of biosurfactant by hydrocarbon degrading Rhodococcus ruber and Rhodococcus erythropolis. Revista de Microbiologia, n. 30, p. 231-236, 1999. 